\title{
The Search for Time-Dependent CP-Asymmetries in the Neutral $B$-Meson System
}

\author{
Michael D. Sokoloff \\ Physics Department \\ University of Cincinnati \\ Cincinnati, OH, U.S.A. \\ E-mail: sokoloff@physics.uc.edu
}

Received 7 January, 2000

\begin{abstract}
In this talk I first present an overview of Standard Model predictions for $C P$ violation in the neutral $B$-meson sector. Then, I present a status report from the $\mathrm{BA} \bar{B} \mathrm{AR}$ experiment at the Stanford Linear Accelerator Center which has been designed especially to measure time-dependent asymmetries whose interpretation in the Standard Model is directly related to the parameters of the Cabibbo-Kobayashi-Maskawa mixing matrix.
\end{abstract}

\section{Introduction}

As particle physicists, we study the fundamental constituents of matter and their interactions. Our understanding of these issues is built upon certain principles: that the laws of physics are the same everywhere, that the laws of physics are the same at all times, that the laws of physics are the same in all inertial reference frames, and that the laws of physics should describe how the wave function of a system evolves in time. The last two issues are embodied in the special theory of relativity and in quantum mechanics. While these principles do not tell us what types of fundamental constituents exist, or how they interact, they restrict the types of theories we consider.

Quantum field theories marry the requirements of special relativity and quantum mechanics, giving us a powerful tool to describe fundamental interactions. A general feature of quantum field theories is that for every type of particle in the theory, there must be a corresponding anti-particle with the same mass and lifetime, and opposite charges (electric, weak, strong, etc.).

We say that a theory is invariant under charge conjugation, $C$, if the laws of physics are precisely the same for a system of anti-particles as for the corresponding system of particles. Similarly, we say that a theory is invariant under parity inversion, $P$, if the laws of physics remain precisely the same under a coordinate transformation that takes $(x, y, z)$ to $(-x,-y,-z)$. Note that parity inversion turns left-handed particles (momentum and helicity anti-parallel) into right-handed particles (momentum and helicity parallel). The weak interaction violates both $C$ and $P$ maximally; that is, it allows only left-handed interactions of particles and only right-handed interactions of anti-particles. However, as a very good approximation, the left-handed weak interactions of particles mimic the right-handed weak interactions of anti-particles. The weak interaction is almost invariant under $C P$.

The electromagnetic and strong nuclear interactions are invariant under $C P$ transformations, and the weak nuclear interaction has been observed to violate $C P$ at a low level $\left(\approx 10^{-3}\right)$, and only in decays of strange mesons which are rare in every day life. However, the universe is observed to be made up overwhelmingly of matter and not of antimatter. Thus, at the most fundamental level we have studied, the interactions of matter and anti-matter are almost the same, yet at the most macroscopic level we see evidence that nature discriminates between matter and anti-matter most powerfully.

\section{The Standard Model}

In the past thirty years, we have developed a Standard Model of particle physics to describe the electromagnetic, weak nuclear, and strong nuclear interactions of constituents in terms of quantum field theories. The prototype for these theories is quantum electrodynamics. Almost 150 years ago, Maxwell replaced the concept of action at a distance with that of fields, and he unified the electric and magnetic forces into a single framework. The electric and magnetic fields are derived from the scalar and vector potentials, which naturally form a Lorentz four-vector, $A^{\mu}$. Classical electrodynamics therefore conforms to the requirement that the laws of physics be the same in all inertial frames. Quantum electrodynamics (QED) quantizes $A^{\mu}$, and 
the corresponding particles, which transfer energy and momentum between electrically charged particles, are the photons.

The fundamental strongly interacting particles are called quarks; they come in six flavors: up $(u)$, down $(d)$, charm $(c)$, strange $(s)$, top $(t)$, and bottom $(b)$. Three $(u, c$, and $t)$ have electric charge $+2 / 3 e$ and are referred to as up-type; the other three $(d, s$, and $b)$ have electric charge $-1 / 3 e$ and are referred to as down-type. The theory of strong interactions, quantum chromodynamics (QCD), has a more complicated charge structure than does QED. Where QED has only one type of charge, which comes in plus or minus, QCD has three types of charge, whimsically called color, each of which comes with its own anti-charge or anti-color. A quark might be red or blue or green; an anti-quark might be anti-red, anti-blue, or anti-green. The quanta of the theory, which transfer energy and momenta between strongly charged particles, are called gluons. They are analogous to the photons of QED. However, unlike photons, which are electrically neutral, gluons carry strong charge, each having color/anti-color quantum numbers such as red/blue-bar.

Quarks have never been observed as free particles; only color-neutral objects seem to exist as asymptotic states. Color neutrality can be created by combining three quarks with different colors to form particles we call baryons, or by combining a quark and an anti-quark of corresponding anti-color to form particles we call mesons. The most common baryons are the proton, which is a uud combination, and the neutron, which is a $u d d$ combination. The most common mesons are the pions which can be neutral or charged. The $\pi^{+}$is a $u \bar{d}$ state. The corresponding strange particle is the $K^{+}$which is a $u \bar{s}$ state. The neutral kaons we observe experimentally are superpositions of $K^{0}$ and $\bar{K}^{0}$ particles which are eigenstates of the weak interaction. The short-lived neutral kaon, the $K_{S}^{0}$, is the symmetric superposition of $\bar{s} d$ and $s \bar{d}$ states. Moving up in mass, the $D^{0}$, a $c \bar{u}$ state, is the lightest charm meson. Similarly, the lightest $B$-meson is the $B^{0}$, a $\bar{b} d$ state. The last meson we will encounter in our survey of $C P$-violation in the neutral $B$ system is the lightest $c \bar{c}$ state, the $\mathrm{J} / \Psi$ particle. Although it has no net charm, is contains equal parts charm and anti-charm and it is produced in decays of both $B$ and $\bar{B}$ mesons.

Weak charged-current interactions differ fundamentally from electromagnetic or strong interactions; the analogues of the photons and gluons associated with this interaction, the $W^{ \pm}$particles, transfer electric charge as well as energy and momentum. A $u$-quark can transform into a $d$-quark by emitting a $W^{+}$, for example. As a first approximation, the weak charged current interaction couples fermions of the same generation: $u$ couples to $d ; c$ couples to $s$; and $t$ couples to $b$ so that the flavor eigenstates (of the strong interaction) correspond to the weak eigenstates. But the weak inter- action does allow transformations across generations.

In the Standard Model, the Cabibbo-KobayashiMaskawa $(C K M)$ matrix, $\mathbf{V}$, transforms the flavor eigenstates to weak eigenstates at the quark level

$$
\left(\begin{array}{c}
d^{\prime} \\
s^{\prime} \\
b^{\prime}
\end{array}\right)=\left(\begin{array}{ccc}
V_{u d} & V_{u s} & V_{u b} \\
V_{c d} & V_{c s} & V_{c b} \\
V_{t d} & V_{t s} & V_{t b}
\end{array}\right)\left(\begin{array}{c}
d \\
s \\
b
\end{array}\right)
$$

After absorbing 5 relative phases in the quark fields, the $C K M$ matrix can be described in terms of three rotation angles and one complex phase $\delta$. It is this (nonzero) phase which leads to $C P$ violation in the Standard Model.

A parameterization introduced by Lincoln Wolfenstein [1] expresses the elements of the CKM matrix in powers of the Cabibbo angle, $\lambda$, and three other real parameters, $A, \rho$, and $\eta$ :

$$
\begin{aligned}
& V_{W}= \\
& \quad\left(\begin{array}{ccc}
1-\frac{1}{2} \lambda^{2} & \lambda & A \lambda^{3}(\rho-i \eta) \\
-\lambda & 1-\frac{1}{2} \lambda^{2}-i A^{2} \lambda^{4} \eta & A \lambda^{2} \\
A \lambda^{3}(1-\rho-i \eta) & -A \lambda^{2} & 1
\end{array}\right) .
\end{aligned}
$$

The phase angle $\delta$ of the PDG parameterization [2] is the arctangent of $\eta / \rho$, and the discussion of the $C P$ violation is often expressed in terms of measurements in the $\rho-\eta$ plane.

In the Standard Model, the $C K M$ matrix is unitary:

$$
\left(\begin{array}{ccc}
V_{u d}^{*} & V_{c d}^{*} & V_{t d}^{*} \\
V_{u s}^{*} & V_{c s}^{*} & V_{t s}^{*} \\
V_{u b}^{*} & V_{c b}^{*} & V_{t b}^{*}
\end{array}\right)\left(\begin{array}{ccc}
V_{u d} & V_{u s} & V_{u b} \\
V_{c d} & V_{c s} & V_{c b} \\
V_{t d} & V_{t s} & V_{t b}
\end{array}\right)=\left(\begin{array}{ccc}
1 & 0 & 0 \\
0 & 1 & 0 \\
0 & 0 & 1
\end{array}\right) .
$$

This leads to a series of "unitarity relationships" which can be described in terms of "unitarity triangles" in the $\rho-\eta$ plane. The most commonly considered relationship is

$$
V_{u b} V_{u d}^{*}+V_{c b} V_{c d}^{*}+V_{t b} V_{t d}^{*}=0
$$

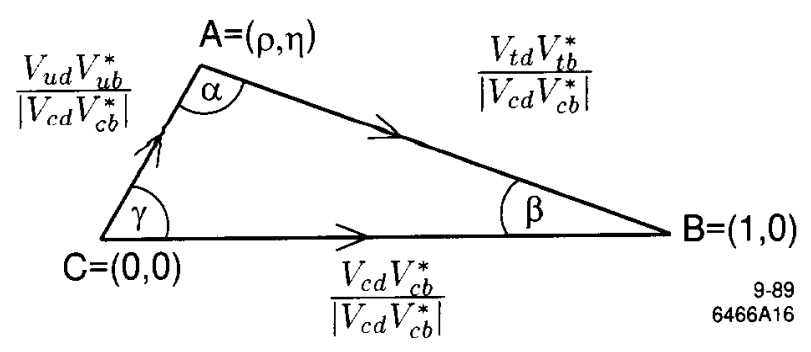

Figure 1. This is the unitarity triangle described in the text. The interior angles $\alpha, \beta$, and $\gamma$ are independent of phase convention.

This is illustrated in Fig. 1 which is reproduced from the $\mathrm{BA} \bar{B}$ AR Technical Design Report (TDR) [3] The interior angles of this triangle, $\alpha, \beta$, and $\gamma$, determine $C P$ violating rates which can be measured experimentally. The interior angles of all the possible unitarity triangles are independent of phase convention in the $C K M$ 
matrix. As is true in the Wolfenstein parameterization, the matrix elements $V_{c d}$ and $V_{c b}$ are usually chosen to be purely real so that the apex of the triangle sits at the point $(\rho, \eta)$. The $C K M$ phase allows for the possibility of $C P$ violation in the weak decays of hadrons when two or more amplitudes contribute to the same final state.

An especially interesting series of measurements can be made in the case of $B^{0}-\bar{B}^{0}$ mixing with subsequent decays to $C P$ eigenstates. Second order weak chargedcurrent processes, often referred to as box diagram amplitudes, provide a mechanism by which $B^{0}$ particles oscillate into $\bar{B}^{0}$ anti-particles, and vice versa. The Feynman diagram for one set of these amplitudes is illustrated in Fig. 2. Because the weak charged current couples generations, the full amplitude must include contributions from all of the up-type quarks coupling to the $b$ quarks in the Feynman diagram. In the limit that these up-type quarks all have the same mass, the unitarity of the CKM matrix leads to exact cancellation of the box diagram amplitudes. Because the quark masses differ, the cancellation is not complete. The top quark mass is so much greater than the charm and up quark masses that the characteristic mixing time in the neutral $B$-meson system is about the same as the characteristic decay time. Writing the equation for mixing as

$$
N_{\text {mix }}=N_{0} e^{-t / \tau} \sin \left(t / t_{\text {mix }}\right),
$$

the experimental result is

$$
t_{\mathrm{mix}} \approx 1.4 \tau \text {. }
$$

Because the characteristic mixing and decay times are so similar, observing the mixing is relatively easy experimentally. Were the mixing time much greater than the decay time, the number of mixed events to observe would be much smaller. And were the mixing time much less than the decay time, current detectors would lack the spatial resolution to resolve the oscillations. (This is the case today for $B_{S}-\bar{B}_{S}$ mixing.)

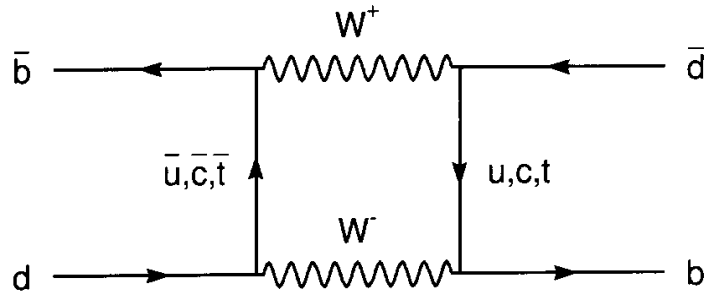

Figure 2. This Feynman diagram represents a series of amplitudes which contribute to $B^{0}-\vec{B}^{0}$ mixing. If all the uptype quark masses were the same, these amplitudes would cancel (assuming the CKM matrix is unitary). Because these masses differ, the cancellation is not complete and the mixing amplitude depends on the top-quark mass and on $V_{t d}$.

The amplitude for $B^{0}$ oscillating into $\bar{B}^{0}$ has a phase equal and opposite to the phase for $\bar{B}^{0}$ oscillating into $B^{0}$. This leads to the expectation for $C P$ violation in $B^{0}-\bar{B}^{0}$ mixing. Consider a $B$-decay final state $f$, such as $J / \Psi K_{S}^{0}$, which is available for either $B^{0}$ or $\vec{B}^{0}$. If a $B$-meson starts life as a $B^{0}$ it may decay into $f$ either directly, or having oscillated into a $\bar{B}^{0}$ first. Similarly, if it starts life as a $\bar{B}^{0}$ it may decay into $f$ directly, or having oscillated into a $B^{0}$ first. The relative phases of the two amplitudes which take $B^{0}$ to $f$, one directly and the other via mixing, will change sign when we consider $\bar{B}^{0}$ to $f$. This leads to asymmetries in the decay rates to $f$. And because the ratio of mixed to unmixed events varies with time, the asymmetry will also vary with time, providing important experimental constraints when interpreting results.

The two amplitudes which interfere are the mixed and unmixed amplitudes $\left(a_{i}\right.$ and $\left.\bar{a}_{i}\right)$ to a $C P$ eigenstate. This $C P$ violation does not depend on the strong phase. Interpretation is especially easy if there is only one $a_{i}$ associated with $B^{0}$ decay to a particular final state. The asymmetries associated with such decays provide direct measurements of the angles $\alpha, \beta$, and $\gamma$.

The algebra is tedious but fairly straight-forward. The time evolution of a $B^{0}$ or $\bar{B}^{0}$ is determined by Schrödinger's Equation:

$$
i \frac{\partial}{\partial t}\left(\begin{array}{c}
\left|B^{0}\right\rangle \\
\left|\vec{B}^{0}\right\rangle
\end{array}\right)=\left[\begin{array}{cc}
M-i \Gamma / 2 & M_{12}-i \Gamma_{12} / 2 \\
M_{12}^{*}-i \Gamma_{12}^{*} / 2 & M-i \Gamma / 2
\end{array}\right]\left(\begin{array}{c}
\left|B^{0}\right\rangle \\
\left|\vec{B}^{0}\right\rangle
\end{array}\right) .
$$

Diagonalizing the Hamiltonian, Schrödinger's Equation has solutions of the form $e^{-i \omega t}=e^{-i(m-i \gamma / 2) t}$ with eigenvalues

$$
m_{H, L}=M \pm R e\left[\left(M_{12}-i \Gamma_{12}\right)\left(M_{12}^{*}-i \Gamma_{12}^{*}\right)\right]
$$

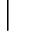

$$
\gamma_{H, L}=\Gamma \mp \operatorname{Im}\left[\left(M_{12}-i \Gamma_{12}\right)\left(M_{12}^{*}-i \Gamma_{12}^{*}\right)\right]
$$

for the "light" and "heavy" eigenstates

$$
\left|B_{L}\right\rangle=p\left|B^{0}\right\rangle+q\left|\bar{B}^{0}\right\rangle ; \quad\left|B_{H}\right\rangle=p\left|B^{0}\right\rangle-q\left|\bar{B}^{0}\right\rangle
$$


with

$\frac{q}{p}=\frac{1+\epsilon_{B}}{1-\epsilon_{B}}=\left(\frac{M_{12}^{*}-i \Gamma_{12}^{*}}{M_{12}-i \Gamma_{12}}\right)^{\frac{1}{2}}=e^{2 i \phi_{M}}\left(\approx \sqrt{\frac{M_{12}^{*}}{M_{12}}}\right)$

The mass and width differences of the eigenstates are usually discussed in terms of the variables

$$
\begin{array}{cc}
\Delta m=m_{H}-m_{L} ; & x=\Delta m / \Gamma \\
\Delta \Gamma=\gamma_{h}-\gamma_{L} ; & y=\Delta \Gamma / 2 \Gamma .
\end{array}
$$

The value of the mass difference variable for the $B_{d}$ system, $x_{d}$, is determined to be $0.73 \pm 0.04$ by the PDG
[2]. No experimental evidence exists for a width difference, and theorists generally expect $y \sim \mathcal{O}\left(10^{-2}\right)$. Thus, the phase of $q / p$ above depends only on $M_{12}$. In the Standard Model, $M_{12}$ originates in a box diagram that generates the phase

$$
2 \phi_{M}=\operatorname{Arg} \sqrt{\frac{M_{12}^{*}}{M_{12}}}=\operatorname{Arg}\left(\frac{V_{t d} V_{t b}^{*}}{V_{t d}^{*} V_{t b}}\right) \rightarrow \operatorname{Arg}\left(\frac{V_{t d}}{V_{t d}^{*}}\right)
$$

where we assume a parameterization of the $C K M$ matrix in which $V_{t b}$ is real.

The flavor eigenstates $\left|B^{0}(t)\right\rangle$ and $\left|\bar{B}^{0}(t)\right\rangle$ can be written in terms of the weak eigenstates:

$$
\begin{aligned}
& \left|B^{0}(t)\right\rangle \\
= & \frac{1}{2 p}\left[\left|B_{L}\right\rangle e^{-\left(\Gamma_{H} / 2+i m_{H}\right) t}+\left|B_{H}\right\rangle e^{-\left(\Gamma_{L} / 2+i m_{L}\right) t}\right] \\
= & e^{-(\Gamma / 2-i m) t}\left[\cos \left(\frac{\Delta m}{2} t\right)\left|B^{0}\right\rangle+\left(\frac{q}{p}\right) i \sin \left(\frac{\Delta m}{2} t\right)\left|\bar{B}^{0}\right\rangle\right] \\
= & \left|\bar{B}^{0}(t)\right\rangle \\
= & \frac{1}{2 q}\left[\left|B_{L}\right\rangle e^{-\left(\Gamma_{H} / 2+i m_{H}\right) t}-\left|B_{H}\right\rangle e^{-\left(\Gamma_{L} / 2+i m_{L}\right) t}\right] \\
= & e^{-(\Gamma / 2-i m) t}\left[\left(\frac{p}{q}\right) i \sin \left(\frac{\Delta m}{2} t\right)\left|B^{0}\right\rangle+\cos \left(\frac{\Delta m}{2} t\right)\left|\bar{B}^{0}\right\rangle\right],
\end{aligned}
$$

where $m \equiv\left(m_{H}+m_{L}\right) / 2$ and $\Gamma=\Gamma_{H}=\Gamma_{L}$.

For any final state $f$, there are four decay amplitudes for the pure $B^{0}$ and $\bar{B}^{0}$ states:

$$
\begin{array}{ll}
\mathcal{A}_{f} \equiv\left\langle f|\mathcal{H}| B^{0}\right\rangle & \overline{\mathcal{A}}_{f} \equiv\left\langle f|\mathcal{H}| \bar{B}^{0}\right\rangle \\
\mathcal{A}_{\bar{f}} \equiv\left\langle\bar{f}|\mathcal{H}| B^{0}\right\rangle & \overline{\mathcal{A}}_{\bar{f}} \equiv\left\langle\bar{f}|\mathcal{H}| \bar{B}^{0}\right\rangle .
\end{array}
$$

The decay rate to a particular final state depends on all the amplitudes which can produce it:

$$
\frac{d \Gamma}{d t} \propto\left|\sum \mathcal{A}\right|^{2}
$$

The time-dependent asymmetry becomes

$$
\begin{aligned}
A(t) & \equiv \frac{d \Gamma / d t-d \bar{\Gamma} / d t}{d \Gamma / d t+d \bar{\Gamma} / d t} \\
& \Rightarrow \frac{\left(1-|\kappa|^{2}\right) \cos (\Delta m t)-2 \sin (\Delta m t) \mathcal{I} m(\kappa)}{1+|\kappa|^{2},}
\end{aligned}
$$

where $\kappa \equiv(q / p)\left(\overline{\mathcal{A}}_{f} / \mathcal{A}_{f}\right)$. For $B^{0}$ and $\bar{B}^{0}$ decays to $C P$ eigenstates in which only one tree-level amplitude contributes,

$$
\frac{\overline{\mathcal{A}}_{f}}{\mathcal{A}_{f}} \rightarrow \frac{\left|A_{t}\right| e^{-i \phi_{t}}}{\left|A_{t}\right| e^{i \phi_{t}}}=e^{-2 i \phi_{t}}
$$

so that the decay asymmetry becomes

$$
A(t) \rightarrow \sin (\Delta m t) \sin 2\left(\phi_{t}-\phi_{M}\right)(-1)^{C P},
$$

where the superscript is the $C P$ eigenvalue of $f$.

Two of the most important $C P$ eigenstates $\mathrm{BA} \bar{B} \mathrm{AR}$ will study are $B^{0} \rightarrow \Psi K_{s}^{0}\left(\mathrm{BR} \approx 5 \times 10^{-4}\right)$, for which [4] (using the Wolfenstein parameterization)

$$
\begin{gathered}
\frac{\overline{\mathcal{A}}_{f}}{\mathcal{A}_{f}} \propto\left(\frac{V_{c b} V_{c s}^{*}}{V_{c b}^{*} V_{c s}}\right)\left(\frac{V_{c s} V_{c d}^{*}}{V_{c s}^{*} V_{c d}}\right) \Rightarrow 2 \phi_{t} \rightarrow 0 \\
\Rightarrow 2\left(\phi_{t}-\phi_{M}\right) \Rightarrow \operatorname{Arg}\left(\frac{V_{t d}^{*}}{V_{t d}}\right)=-2 \beta,
\end{gathered}
$$

and $B^{0} \rightarrow \pi^{+} \pi^{-}\left(\mathrm{BR} \approx 2 \times 10^{-5}\right)$, for which $[4]$

$$
\begin{aligned}
& \frac{\overline{\mathcal{A}}_{f}}{\mathcal{A}_{f}} \propto\left(\frac{V_{u b}^{*} V_{u d}}{V_{u b} V_{u d}^{*}}\right) \Rightarrow 2 \phi_{t} \rightarrow \operatorname{Arg}\left(\frac{V_{u b}^{*}}{V_{u b}}\right) \\
& \Rightarrow 2\left(\phi_{t}-\phi_{M}\right) \rightarrow \operatorname{Arg}\left(\frac{V_{u b}^{*} V_{t d}^{*}}{V_{u b} V_{t d}}\right)=2 \alpha,
\end{aligned}
$$

assuming only tree-level amplitudes are significant. For $B^{0} \rightarrow \Psi K_{s}^{0}$, the penguin amplitudes have the same 
weak phase as does the tree-level amplitude, so measuring the asymmetry still allows the direct extraction of $\sin 2 \beta$. For $B^{0} \rightarrow \pi^{+} \pi^{-}$the penguin and tree-level weak phases differ, so extracting $\sin 2 \alpha$ from the asymmetry measurement will require more experimental and theoretical work [4].

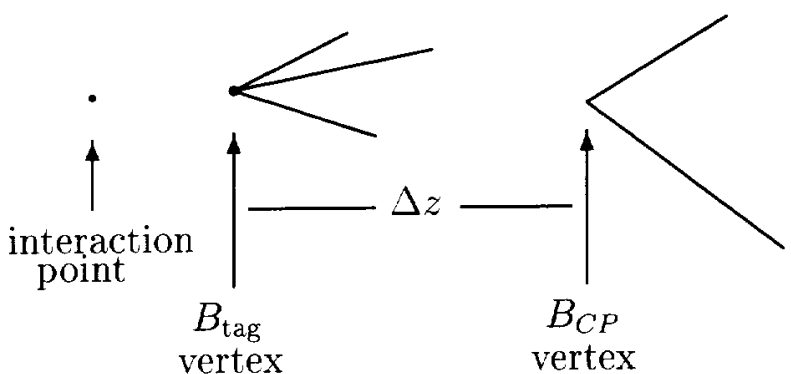

Figure 3. This cartoon illustrates the relationship between the $e^{+} e^{-}$interaction point and the decay points of the two $B$-mesons used in measuring time-dependent $C P$ violation. Because the $B$-mesons are produced approximately at rest in the $\mathrm{CM}$, the vertex separation is primarily along the direction of motion of the $\mathrm{CM}$.

Experimentally, $\Delta M \times \Gamma \approx 0.7$, which makes it possible to observe the time dependencies of these asymmetries. BA $\bar{B}$ AR is doing this using asymmetric $e^{+} e^{-} \rightarrow$ $\Upsilon(4 S) \rightarrow B^{0} \bar{B}^{0}$ events, as illustrated in Fig. 3. The

\section{$\vec{B}^{0}$ Tagged}

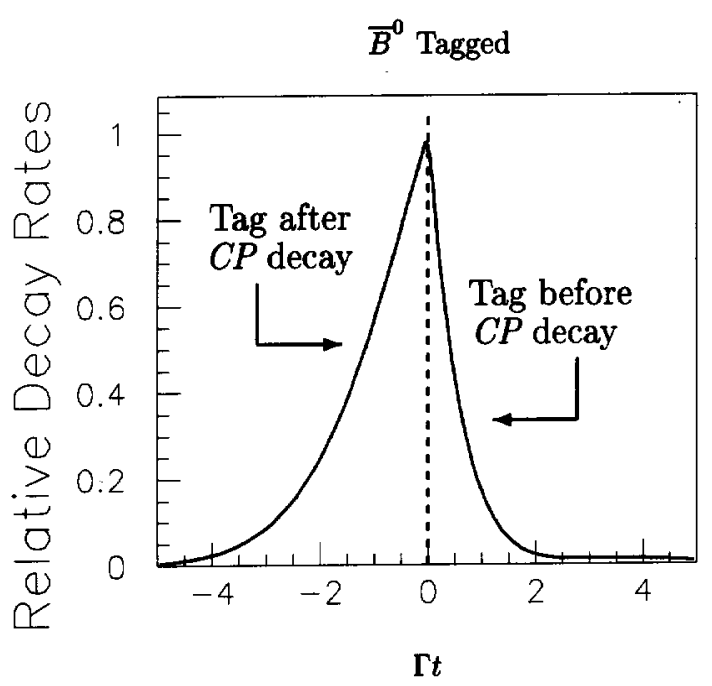

high energy $\left(e^{-}\right)$ring operates at $9.0 \mathrm{GeV}$. The low energy $\left(e^{+}\right)$ring operates at $3.1 \mathrm{GeV}$. Because the mass of the $\Upsilon(4 S)$ is just above threshold for the production of $B \bar{B}$ pairs, the $B$-mesons as well as the $\Upsilon(4 S)$ are co-moving along the beam axis with $\beta \gamma=0.56$. The typical separation between the two $B$ decay vertices in an event is $250 \mu \mathrm{m}$.

Because the $\Upsilon(4 S)$ is a pure $(C P=-1)$ state, the $B^{0} \bar{B}^{0}$ state is coherent until one of the $B^{\prime}$ 's decays. At this point, one is a $B^{0}$ and the other a $\bar{B}^{0}$. For the evolution equations presented earlier, this defines time $t=0$. When one $B$ decays to a $C P$ eigenstate, denoted as the $B_{C P}$ decay, and the other is tagged as $B^{0}$ or $\bar{B}^{0}$, denoted as $B_{\text {tag }}$, the decay time $t$ is measured from the distance of the decay point of $B_{\text {tag }}$ to the decay point of $B_{C P}(\Delta z)$. The asymmetries for $\Delta z<0$ should be equal and opposite to those for $\Delta z>0$. Measurement errors on $\Delta z$ depend on both the $B_{\text {tag }}$ and $B_{C P}$ vertex measurements. In $\mathrm{BA} \bar{B} \mathrm{AR}$, the convoluted $\sigma(\Delta z)$ is typically $<80 \mu \mathrm{m}$, which is small enough compared to the typical vertex separation that it degrades $C P$ violation measurements only modestly. The relative decay rates for $B^{0}$ and $\bar{B}^{0}$ decay to a $C P$ eigenstate whose tree-level amplitude depends only on the unitarity angle $\beta$ are illustrated in Fig. 4 for nominal values of mass difference and $\beta$.

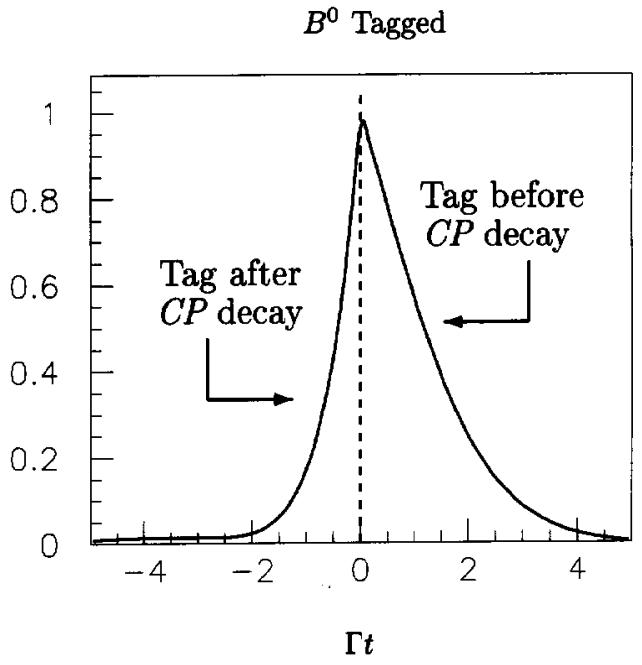

Figure 4. The relative decay rates, $\Gamma\left(B_{C P}\right) \propto e^{-\Gamma|t|}[1 \mp \sin 2 \beta \sin (\Delta m t)]$, for events which "start" life as $B^{0}(-)$ and $\bar{B}^{0}$ $(+)$ assuming $\beta=0.5 ; \Delta m / \Gamma=0.7$.

In the Standard Model, the unitarity angles $\alpha, \beta$ and $\gamma$ are determined by the parameters $\rho$ and $\eta$. These are constrained by data from $K^{\circ}$ decay $(\epsilon), B$ and charm decays, and from $B_{d}^{\circ}-\overline{B_{d}^{\circ}}$ mixing. In addition, theoretical calculations of hadronic matrix elements are used. The shaded area in Fig. 5, taken from the review article by Richman and Burchat [5], corresponds to that allowed for the apex of the unitarity triangle circa 1995.
These measurements indirectly constrain the phase the of $C K M$ matrix. But they do not establish the existence of the phase or establish the unitarity of the $C K M$ matrix. Rather, they assume these features. Measuring the time-dependent $C P$ asymmetries in $B^{0}$ and $B_{S}$ decay can directly establish the $C K M$ phase as the origin of $C P$ violation. Checking experimentally that $\alpha+\beta+\gamma$ $=180^{\circ}$ will test the unitarity of the $C K M$ matrix. Comparing the angles derived by these $C P$ asymmetry mea- 
surements with those determined from ratios of branching ratios, the $B^{0}-\bar{B}^{0}$ mixing rate, etc. will provide further tests of the Standard Model.

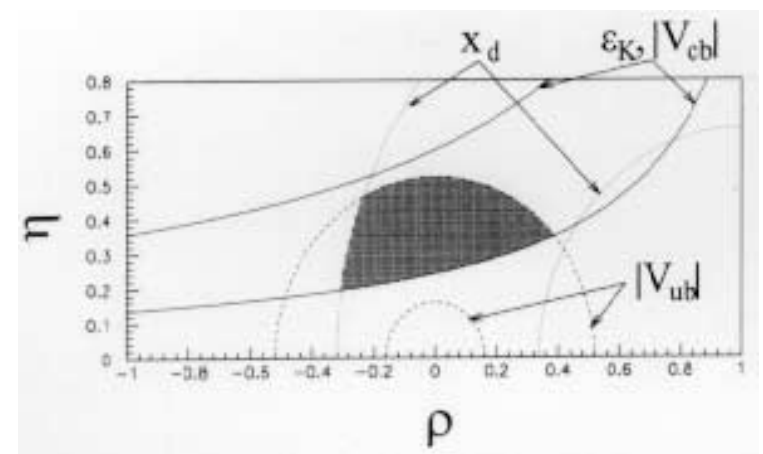

Figure 5. Constraints in the Standard Model in the $\rho \eta$ plane. The shaded area corresponds to that allowed for the apex of the rescaled unitarity triangle circa 1995 [5].

\section{The $\mathrm{BA} \bar{B} \mathrm{AR}$ Experiment}

The BA $\bar{B}$ AR experiment has been built at the Stanford Linear Acceleration Center (SLAC) to exploit PEPII, an asymmetric $e^{+} e^{-}$collider which operates at the $\Upsilon(4 s)$ resonance. At the peak of this resonance, approximately $25 \%$ of all hadronic events are $B \bar{B}$ events, and of these, approximately half are $B^{0} \bar{B}^{0}$. Shortly after turning on the detector in the beamline, we ran an energy scan to find the location of the resonance. The rate of multi-hadron events is plotted as a function of energy in Fig. 6. The curve plotted on top of the data is the theoretically expected shape fitted to the data. Although the agreement is good, the final analysis remains to be done, so no quantitative results are available yet.

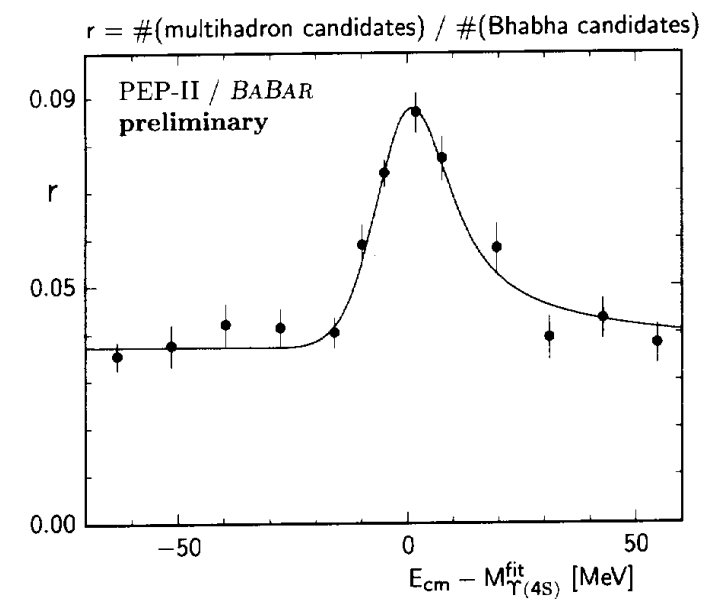

Figure 6. Energy scan data from early summer, 1999.

BA $\bar{B}$ AR has five major detector systems. Just outside the beam pipe is a silicon vertex tracker which provides the precision measurements of vertex separation in the time-dependent asymmetry studies which the experiment is designed to make. This is surrounded by a cylindrical drift chamber which has 40 layers. In addition to providing tracking information, measurements of the specific ionization allow particle identification over a useful momentum range. Surrounding the drift chamber is the DIRC, a novel ring-imaging Cerenkov detector whose radiators are quartz bars. This is surrounded by a CsI electromagnetic calorimeter. All these elements of the detector sit inside a superconducting solenoid which provides a $1.5 \mathrm{~T}$ field. The iron flux return is instrumented to detect muons and neutral hadrons.
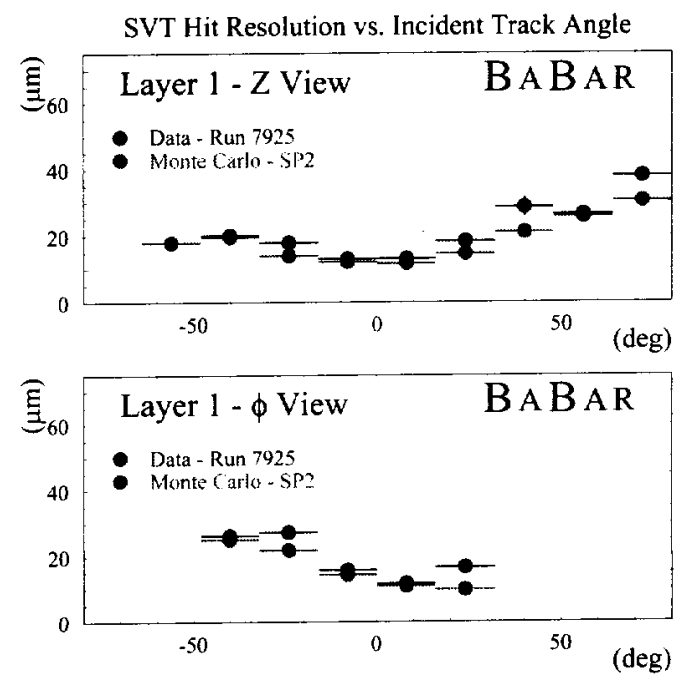

Figure 7. This figure shows the azimuthal and longitudinal resolutions in the first layer of the SVT as a function of track angle measured using cosmic ray data before beam was available. Monte Carlo data is compared to real data, showing that the detector performance is approaching design specifications.

The silicon vertex tracker has five layers of doublesided silicon microstrip detectors. One side measures the longitudinal coordinate, the other measures the azimuthal coordinate. In cosmic ray data taken before collisions started in the detector, the azimuthal and longitudinal resolutions were measured with r.m.s. values of $15 \mu \mathrm{m}$ and $19 \mu \mathrm{m}$, as observed in Fig. 7. The resolution varies as a function of the angle at which a track traverses the detector. Fig. 7 shows how the resolution in the first layer of the SVT varies as a function of laboratory angle for a single run, and compares the measurements with Monte Carlo simulations. 


\section{Drift Chamber Hit Resolution}

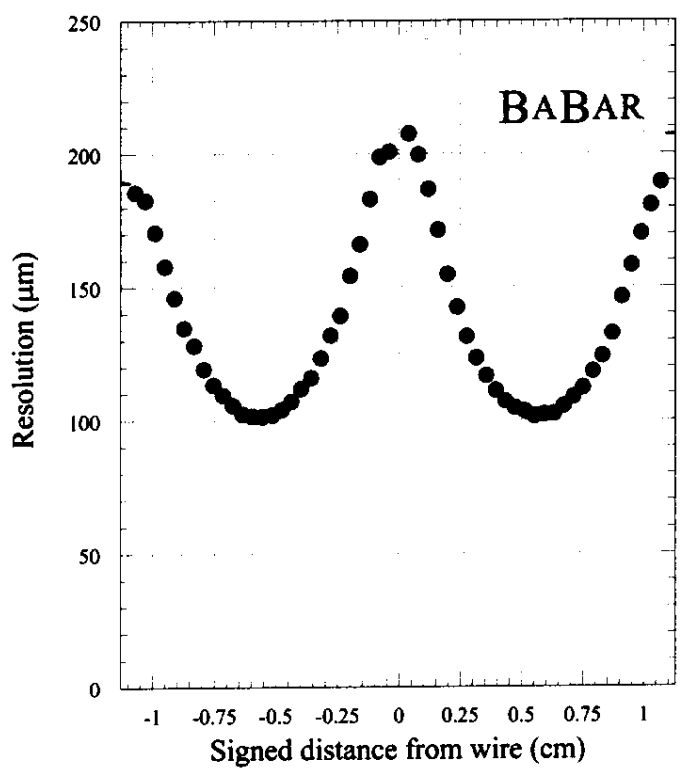

Figure 8. Drift chamber resolution is shown as a function of track position within a drift chamber cell. Most tracks occupy regions where the resolution is good, so the average resolution is now $125 \mu \mathrm{m}$ where the design value was 140 $\mu \mathrm{m}$.

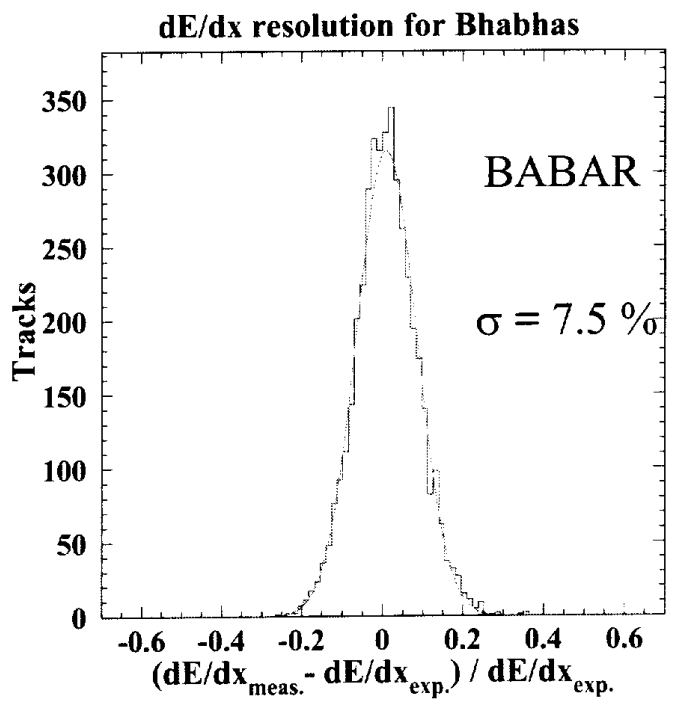

Figure 9. Specific ionization in the drift chamber from Bhabha scattering events. The design resolution is $7 \%$, so once again we see that the detector performance is rapidly approaching design specifications.

The drift chamber has 40 layers divided into 10 alternating axial and stereo superlayers. We use a low density $80 \% \mathrm{He}, 20 \%$ isobutane gas mixture and aluminum wires. The detector is designed to provide $7 \%$ ionization $(d E / d x)$ resolution and $140 \mu \mathrm{m}$ spatial resolution. Fig. 8 shows the measured spatial resolution as a function of position in a drift chamber cell. The mean value, weighted for how many tracks traverse each part of a cell, is $125 \mu \mathrm{m}$, which is better than the design specification. The $d E / d x$ resolution measured in Bhabha events is shown in Fig. 9. The resolution is about $7.5 \%$, which is approaching the design resolution. This will allow good $\pi$-K separation up to $700 \mathrm{MeV} / c$, and good proton identification up to $1.2 \mathrm{GeV} / c$, as seen in Fig. 10.

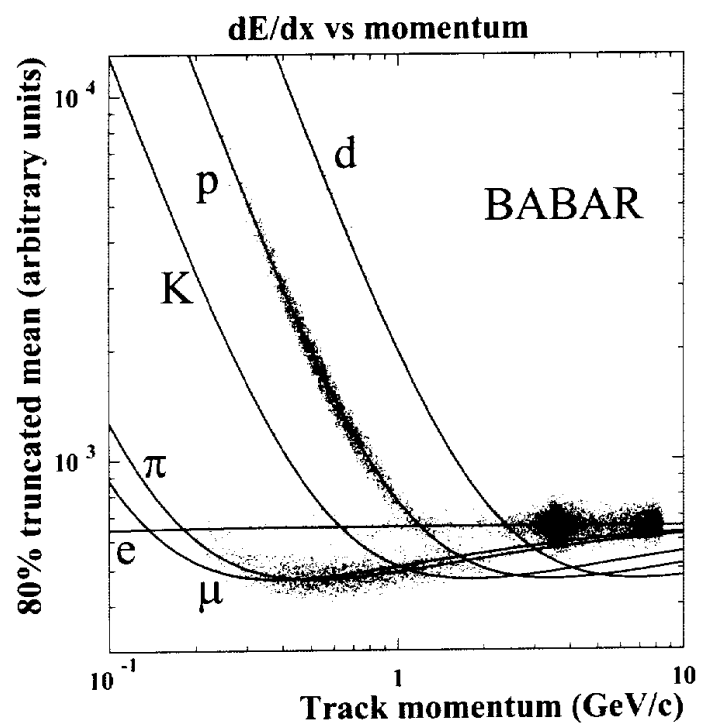

Figure 10. Specific ionization measured in the drift chamber in early summer, 1999. Good $\pi / K$ separation is observed below $600 \mathrm{MeV} / c$.

The DIRC is a detector of internally reflected Cerenkov light. Its principle of operation is illustrated in Fig. 11. When a fast charged track traverses a quartz bar (index of refraction $\approx 1.47$ ), it emits Cerenkov light in a cone coaxial with the track's motion. Most of the photons will be totally internally reflected at the interface of the quartz bar and air, and will be transported to a water standoff box where they will be refracted rather than reflected. The image of the cone expands as the photons pass through $1.2 \mathrm{~m}$ of water before impinging on an array of photomultiplier tubes. The angle of an individual photon with respect to the charged track is determined by tracing its trajectory backwards, assuming it emerged from the center of the quartz bar and hit the center of the phototube. The cone of light emitted by a single track will transform into a conic section in the array of phototubes, and the Cerenkov angle associated with each track is determined by fitting the Cerenkov angles of the single photons which might be part of its image. 


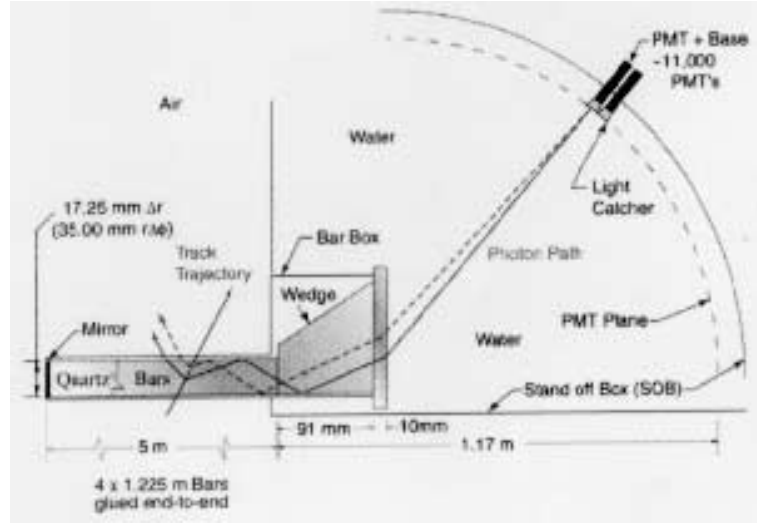

Figure 11. The DIRC measures the Cerenkov angle of light produced by charged particles traversing quartz bars to identify the species of the charged particles. Photons are internally reflected to the end of the quartz bars where they emerge into a water stand-off box and spread out forming conical images on the plane of phototubes.

The Cerenkov angles for backward positrons from Bhabha events is shown in Fig. 12. The observed resolution is $3.0 \mathrm{mrad}$. The design resolution for such tracks is $2.6 \mathrm{mrad}$. This detector is rapidly approaching its design specification.

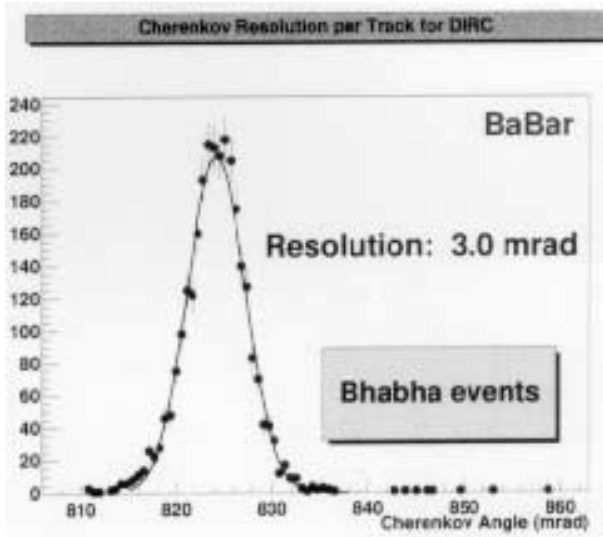

Figure 12. This figure shows the Cerenkov angle measured for tracks from Bhabha scattering events. The observed resolution is about $3.0 \%$ where the design specification is $2.6 \%$.

The electromagnetic calorimeter is a critical detector for time-dependent $C P$-asymmetry measurements. The standard gold-plated channel is $B$ to $J / \Psi K_{S}^{0}$, where $\Psi \rightarrow \ell^{+} \ell^{-}$. Furthermore, tagging the non- $C P B$ is done most frequently and most cleanly with high $p_{T}$ leptons. In addition, it allows us to identify $\pi^{0} \rightarrow \gamma \gamma$ and $\eta \rightarrow \gamma \gamma$ candidates for use in reconstructing $B$ and $D$ decays. A clear $\pi^{0}$ peak is observed in the $\gamma \gamma$ invariant mass spectrum shown in Fig. 13.

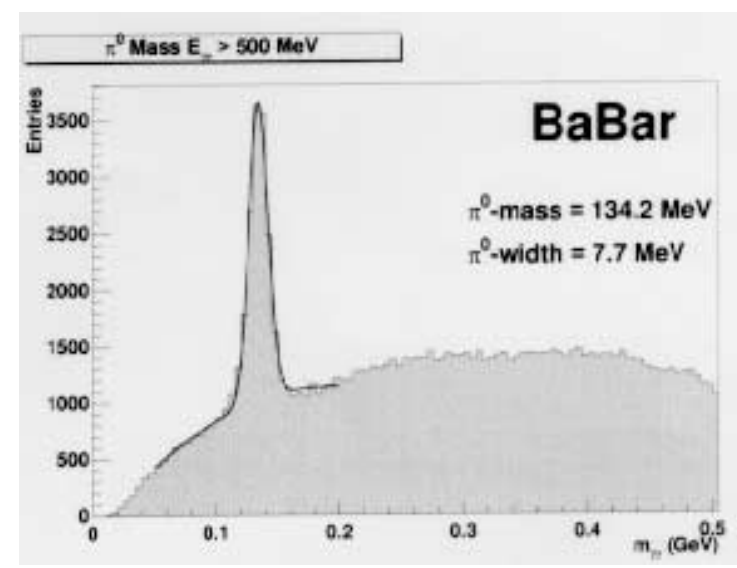

Figure 13. A clear $\pi^{0}$ peak emerges when we require each photon have energy greater than $50 \mathrm{MeV}$ and the di-photon pair have energy greater than $500 \mathrm{MeV}$.

To search for $B \rightarrow J / \Psi K_{S}^{0}$, we first look at inclusive distributions to see the constituents. Fig. 14 shows a clean inclusive $K_{S}^{0}$ peak in a $\pi^{+} \pi^{-}$invariant mass distribution. The two tracks were required to form a good vertex, and the summed momentum vector was required to point back to the beam spot. Fig. 15 shows inclusive $e^{+} e^{-}$and $\mu^{+} \mu^{-}$distributions in which we see $J / \Psi$ signals. The data are very much preliminary, but the efficiencies are roughly what we expect at this stage of the experiment.

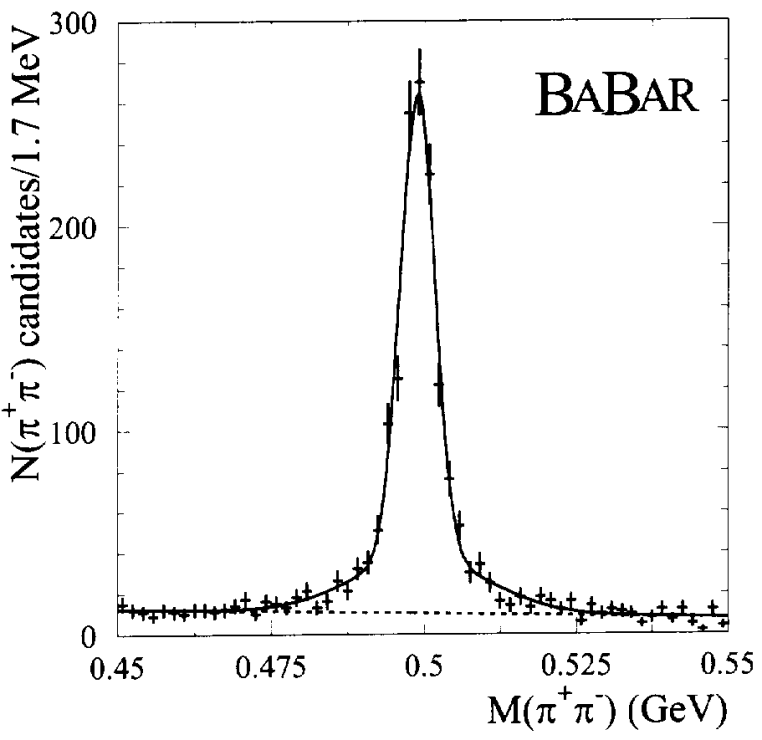

Figure 14. kshort caption

To select candidate $B \rightarrow J / \Psi K_{S}^{0}$ combinations, we calculate the invariant mass of four-track candidates where the constituents form $J / \Psi$ and $K_{S}^{0}$ candidates consistent with coming from a common vertex. We calculate the invariant mass of the $J / \Psi K_{S}^{0}$ candidate, and also the difference between this combination's energy in the $e^{+} e^{-}$center-of-mass and the energy expected for 
a $B$-meson produced there, $\Delta E$. The masses and energy differences for the candidates from very early data are plotted in Fig. 16. The box in the scatter plot spans \pm 3 standard deviations in $\Delta E$ and in invariant mass. It occupies about $10 \%$ of the area of the scatter plot. Two entries are observed outside the box, leading to an estimate of roughly 0.2 background events inside the box. Two events are observed. Based on crude luminosity measurements and our current understanding of reconstruction efficiencies, we expect somewhere between 1 and 2 signal events. 4The data is therefore consistent with our expectations and with our having observed at least one $B \rightarrow J / \Psi K_{S}^{0}$ decay. We see similar evidence for $B^{0} \rightarrow D^{*-} \pi^{+}$(another 2 events inside the signal region where background is estimated to be a small fraction of an event). This gives us confidence that the detector is working and our goals for the first year of running are realistic.

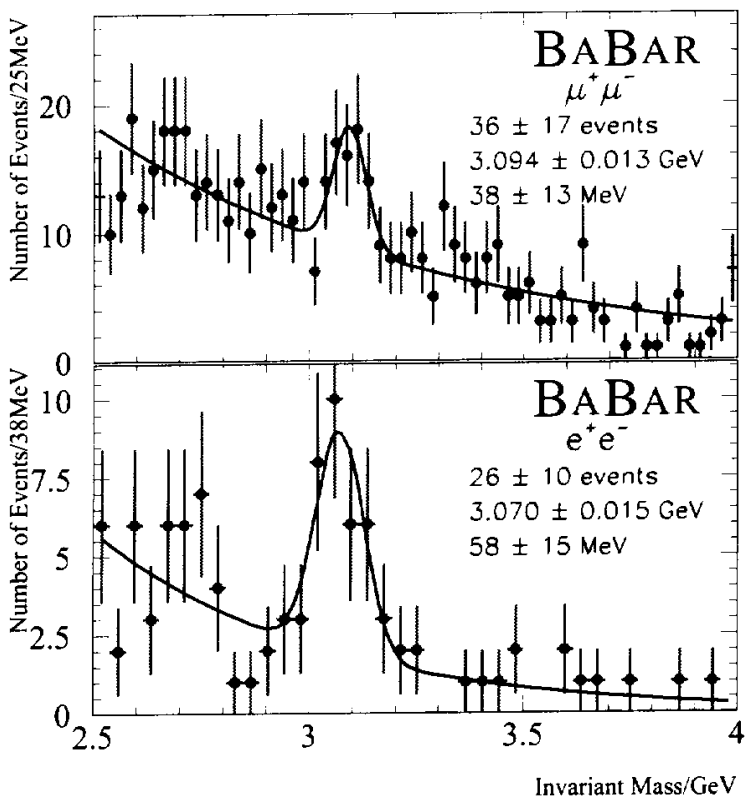

Figure 15. $J / \Psi$ data from early summer, 1999.

The design luminosity of PEP-II is $3 \times$ $10^{33} \mathrm{~cm}^{-2} \mathrm{sec}^{-1}$, which should produce $30 \times 10^{6} \bar{b} b$ pairs per year. At the time of this conference, $\mathrm{BA} \bar{B} \mathrm{AR}$ had recorded more than $1 \mathrm{fb}^{-1}$ of data and PEP-II had achieved a peak luminosity of $1 \times 10^{33} \mathrm{~cm}^{-2} \mathrm{sec}^{-1}$; by the end of 1999 it had recorded almost $2 \mathrm{fb}^{-1}$ of data and achieved a peak luminosity of $1.5 \times 10^{33} \mathrm{~cm}^{-2} \mathrm{sec}^{-1}$. We hope to collect $10 \mathrm{fb}^{-1}$ of data by the summer of 2000. With $10 \mathrm{fb}^{-1}$ of data we should be able to reconstruct $200 B \rightarrow J / \Psi K_{S}^{0}$ events and determine the value of $\sin 2 \beta$ with a precision of 0.2 , sufficient for a 3 standard deviation measurement, if the central value is close to that expected.

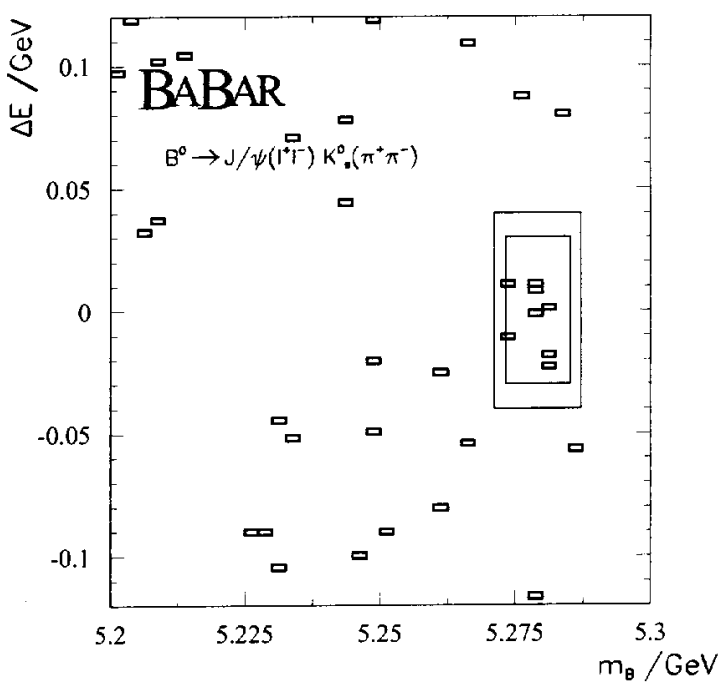

Figure 16. For each $\Psi K_{S}^{0}$ candidate, the invariant mass and the difference between expected and observed center-ofmass energies are calculated. The boxes around the signal region indicate three standard deviation ranges for candidates with $\Psi$ decaying to electrons or muons. The luminosity for this sample is approximately $435 \mathrm{pb}^{-1}$. The signal region contains 8 entries where just under 1 background entry is expected. The yield is consistent with our expectations given the measured reconstruction efficiencies for this data set.

Over the next three to five years, BA $\bar{B}$ AR should be able to measure $C P$ violation using high statistics samples in many decay modes (thousands in $B \rightarrow J / \Psi K_{S}^{0}$ and thousands in other decay modes). This should allow us to test the Standard Model: if the value of $\sin 2 \beta$ we determine does not accord with the range allowed in the Standard Model by other types of measurements, as illustrated in Fig. 5, we will have evidence of new physics; if the value of $\sin 2 \beta$ measured in one decay mode does not accord with that measured in another decay mode, we will have evidence of new physics; If the values of $\alpha, \beta$, and $\gamma$ that we determine are not consistent with $\alpha+\beta+\gamma=180^{\circ}$, then we will have evidence of new physics. And if all of our measurements are selfconsistent within the framework of the Standard Model, we will have made precision measurements of the relative phases of the $C K M$ matrix elements. Whatever the outcome, our data will help guide the quantitative understanding of $C P$ violation.

It is my pleasure to thank the organizers of this conference for inviting me to participate and to present a status report on the $\mathrm{BA} \bar{B} \mathrm{AR}$ experiment. As is true so often today in experimental high energy physics, the success of the project is the result of very many people working very hard, and my part in the experiment has been very small. In addition to the hundreds of participants in BA $\bar{B}$ AR itself, we are indebted to all those who made the PEP-II accelerator work so well, so quickly, and who continue to push its limits. They are heroes. This work was supported in part by the U.S. National Science Foundation. 


\section{References}

[1] L. Wolfenstein, Parameterization of the KobayashiMaskawa matrix, Phys. Rev. Lett. 51, 1945 (1983).

[2] Particle Data Group, C. Caso et al., Review of Particle Properties, The European Physical Journal C 3, 1 (1998).

[3] The BA $\bar{B}$ AR Collaboration, B. Boutigny et al. , BA $\bar{B}$ AR Technical Design Report, SLAC-R-95-457 (March,
1995).

[4] Y. Nir and H. Quinn, $C P$ violation in $B$ physics, Ann. Rev. Nucl. Part. Sci. 42, 211 (1992), and references therein

[5] Jeffrey D. Richman and Patricia R. Burchat, Leptonic and semileptonic decays of charm and bottom hadrons, Rev. Mod. Phys. 67, 893 (1995). 